Article

\title{
An Established HPLC-MS/MS Method for Evaluation of the Influence of Salt Processing on Pharmacokinetics of Six Compounds in Cuscutae Semen
}

\author{
Jiao Liu ${ }^{1,2,+}$, Shuhan Zou ${ }^{1,2,+}$, Wei Liu ${ }^{1,2}$, Jin $\mathrm{Li}^{1}$, Hui Wang ${ }^{1,2}$, Jiao Hao ${ }^{1,2}$, Jun He ${ }^{1,2}$, \\ Xiumei Gao ${ }^{1,2}$, Erwei Liu ${ }^{1,2, *}$ and Yanxu Chang 1,2,*(D) \\ 1 Tianjin State Key Laboratory of Modern Chinese Medicine, Tianjin University of Traditional Chinese \\ Medicine, Tianjin 300193, China \\ 2 Tianjin Key Laboratory of Phytochemistry and Pharmaceutical Analysis, Tianjin University of Traditional \\ Chinese Medicine, Tianjin 300193, China \\ * Correspondence: liuerwei628@hotmail.com (E.L.); tcmcyx@126.com (Y.C.); \\ Tel.: +86-22-5959-6163 (E.L. \& Y.C.); Fax: +86-22-5959-6163 (E.L. \& Y.C.) \\ + These authors contributed equally to this work.
}

Received: 16 May 2019; Accepted: 2 July 2019; Published: 9 July 2019

check for updates

\begin{abstract}
A sensitive and effective method was developed for clarifying the pharmacokinetic properties of six compounds (including hyperin, chlorogenic acid, neochlorogenic acid, p-coumaric acid, astragalin, and isoquercitrin) in two processed Cuscutae Semen samples by high performance liquid chromatography mass spectrometry (HPLC-MS/MS). The six compounds were separated by acetonitrile and $0.1 \%$ formic acid-water on an Agilent Eclipse plus C18 column $(4.6 \mathrm{~mm} \times 100 \mathrm{~mm}$, $1.8 \mu \mathrm{m})$. All compounds were analyzed with negative ion mode in multiple reaction monitoring (MRM). The lower limits of quantification (LLOQ) of hyperin, astragalin, neochlorogenic acid, chlorogenic acid, isoquercitrin, and p-coumaric acid were $1,0.1,4,0.1,2$, and $4 \mathrm{ng} \cdot \mathrm{mL}^{-1}$, respectively. The validated approach was effectively used for the pharmacokinetics of six compounds of two processed Cuscutae Semen samples after oral administration to rat. The results indicated that salt processing could improve the adsorption and bioavailability of astragalin in Cuscutae Semen.
\end{abstract}

Keywords: Cuscutae Semen; salt processed; HPLC-MS/MS; pharmacokinetic study

\section{Introduction}

Cuscutae Semen (CS) derived from the dry and mature seeds of Cuscuta australis R.Br. or Cuscuta chinensis Lam. is named TuSiZi in Chinese [1]. It is used to nourish the kidney, improve sexual function, treat impotence, prevent miscarriage, and restrain diarrhea $[2,3]$. CS contains some chemical components, including flavonoids, chlorogenic acids, alkaloids, and polysaccharides [4,5]. CS possesses many biological activities, such as anti-inflammatory, neuroprotective, and antinociceptive effects [6,7]. In recent years, some research has indicated that flavonoids and chlorogenic acids are the main active ingredients in CS for anti-inflammatory and antioxidant effects, for preventing miscarriage, and for cardiovascular activities [8-11].

Processing of traditional Chinese medicine (TCM) is a pharmaceutical method to satisfy the needs of treatment. According to the theory of TCM, medicinal materials need special processing methods, such as stir-frying, steaming, calcining, or boiling, which aim to increase effects and decrease toxicity. According to Chinese pharmacopoeia [2], there are two different processing methods of CS, including the raw Cuscutae Semen (R-CS) processed by stir frying and stir frying with salt-water, named 
the stir-frying Cuscutae Semen (SF-CS), and salt-processed Cuscutae Semen (SP-CS), respectively. The SF-CS and SP-CS have different pharmacological properties and are named qing-chao-tu-si-zi and yan-zhi-tu-si-zi in Chinese, respectively. when the medicinal materials are processed, the chemical compounds change, including the increase or decrease of content and the formation or disappearance of compounds. According to the basic theories of TCM, medicinal materials processed with salt can improve the function of nourishing the kidney [12]. The previous report indicated that the contents of quercetin and total compounds increased and hyperoside decreased after salt processing. SP-CS also has enhanced levels of testosterone and antioxidant effects from a biological activity aspect [13]. However, the work mechanism of activity change was not clear after salt processing in vivo.

Pharmacokinetics is an effective method of evaluating the safety of medicine and to clarify the influence of processing on the components absorbed into the body after oral administration in rats [14]. At present, there are only a few reports for pharmacokinetic studies of two compounds (hyperoside and quercetin) after oral administration of Cuscutae Semen extract $[15,16]$. Firstly, the pharmacokinetic information of the two compounds did not clarify the metabolic regularity of the whole herbs in vivo. Next, there has been no report comparing the pharmacokinetic studies of two processing products for CS. Therefore, it is necessary to perform the pharmacokinetics of multiple flavonoid ingredients to clarify the work mechanism of activity change by comparing the pharmacokinetic properties of two processing procedures for CS.

In the present research, an efficient and validated method was developed for simultaneous quantification of six compounds, including hyperin, chlorogenic acid, neochlorogenic acid, p-coumaric acid, astragalin, and isoquercitrin (Figure 1) in pharmacokinetics of CS. Furthermore, the study of multiple compounds in rat plasma were measured in the pharmacokinetic study of two processing products for CS. The established method was effectively used to provide the pharmacokinetic outlines for clinic research of salt-processed CS.<smiles>Cc1ccc(-c2oc3cc(O)cc(O)c3c(=O)c2OC2OC(CO)C(O)C(C)C2C)cc1O</smiles>

Hyperoside<smiles>CC1C(O)C(O)C(CO)C(O)C1Oc1c(-c2ccc(O)cc2)oc2cc(O)cc(O)c2c1=O</smiles>

Astragalin

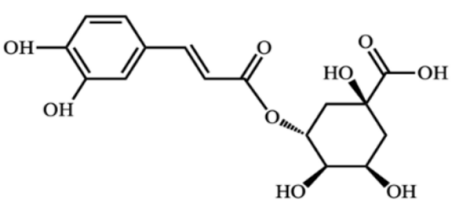

Chlorogenic acid<smiles>O=C1c2c(O)cc(O)cc2OC(c2ccc(O)c(O)c2)C1OC1CC(O)C(O)C(O)C1O</smiles>

Isoquercitrin<smiles>[2H][C@@]1(C(=O)O)C[C@H](O)[C@@H](O)[C@@H](OC(=O)/C=C/c2ccc(O)c(O)c2)C1</smiles>

Neochlorogenic acid<smiles>O=C(O)C=Cc1ccc(O)cc1</smiles>

P-Coumaric acid<smiles>COc1ccc(-c2coc3cc(O)ccc3c2=O)cc1</smiles>

Formononetin

Figure 1. Chemical structures of hyperoside, chlorogenic acid, neochlorogenic acid, astragalin, isoquercitrin, P-Coumaric acid, and formononetin (IS).

\section{Results}

\subsection{Internal Standard (IS) Selection}

In the study, the three kinds of compounds and the three kinds of phenolic acids were simultaneously determined. Formononetin was selected as the internal standard for both flavonoid and phenolic acid owing to the similar structure and it showing no interference from endogenous substances. 


\subsection{Optimization of LC-MS/MS Conditions}

In terms of MS conditions, the responses of analytes were better in multiple-reaction monitoring (MRM) with the negative ion mode. The optimized MS conditions are shown in Table 1. When acetonitrile and $0.1 \%$ formic acid-water were chosen with a flow rate of $0.5 \mathrm{~mL} \cdot \mathrm{mL}^{-1}$, the six compounds obtained a better separation and higher response.

Table 1. The source parameters of the six compounds and internal standard (IS).

\begin{tabular}{ccccccccc}
\hline Compounds & $\mathbf{Q}_{\mathbf{1}}$ & $\mathbf{Q}_{\mathbf{3}}$ & Dwell Time $(\mathbf{m s})$ & $\mathbf{D P}(\mathbf{V})$ & $\mathbf{E P}(\mathbf{V})$ & $\mathbf{C E}(\mathbf{e V})$ & $\mathbf{C X P}(\mathbf{V})$ & Retention (min) \\
\hline Neochlorogenic acid & 353.0 & 190.9 & 100 & -40 & -4.5 & -30 & -3.0 & 3.01 \\
Chlorogenic acid & 353.0 & 191.0 & 100 & -40 & -5.5 & -25 & -1.0 & 3.83 \\
P-coumaric acid & 162.8 & 119.0 & 100 & -35 & -5.5 & -20 & -2.0 & 10.07 \\
Hyperin & 463.0 & 300.0 & 100 & -70 & -6.0 & -39 & -1.0 & 12.51 \\
Isoquercitrin & 462.9 & 300.0 & 100 & -60 & -7.0 & -40 & -1.5 & 13.61 \\
Astragalin & 447.1 & 284.0 & 100 & -68 & -5.5 & -38 & -1.0 & 15.81 \\
Formononetin (IS) & 267.0 & 251.8 & 100 & -60 & -2 & -27 & -16 & 19.25 \\
\hline
\end{tabular}

\subsection{Quantification of the Six Compounds in Two Processed CS Extracts}

According to the extraction yield, the contents of the six compounds for the two processed CS were analyzed. The oral concentrations of hyperin, chlorogenic acid, neochlorogenic acid, p-coumaric acid, astragalin, and isoquercitrin were $21.8,31.1,6.09,0.59,1.87$, and $1.98 \mathrm{mg} \cdot \mathrm{kg}^{-1}$ for SF-CS extracts, respectively, and were $27.5,49.0,6.64,0.87,1.30$, and $1.99 \mathrm{mg} \cdot \mathrm{kg}^{-1}$ for SP-CS extracts, respectively.

\subsection{Method Validations}

\subsubsection{Linearity and LLOQ}

The LLOQ, correlation coefficients and equations of the calibration curves of the six compounds are listed in Table 2. The linearity range was 1 to $250 \mathrm{ng} \cdot \mathrm{mL}^{-1}$ for hyperin, from 0.1 to $1500 \mathrm{ng} \cdot \mathrm{mL}^{-1}$ for chlorogenic acid, 4 to $1000 \mathrm{ng} \cdot \mathrm{mL}^{-1}$ for neochlorogenic acid, 4 to $10,000 \mathrm{ng} \cdot \mathrm{mL}^{-1}$ for $\mathrm{p}$-coumaric acid, 0.1 to $25 \mathrm{ng} \cdot \mathrm{mL}^{-1}$ for astragalin, and 2 to $500 \mathrm{ng} \cdot \mathrm{mL}^{-1}$ for isoquercitrin. Each correlation coefficient was greater than 0.9990 ( $\mathrm{r} \geq 0.9990)$. The lower limits of quantification (LLOQ) of hyperin, neochlorogenic acid, p-coumaric acid, chlorogenic acid, astragalin, and isoquercitrin was 1, 4, 4, 0.1, 0.1, and $2 \mathrm{ng} \cdot \mathrm{mL}^{-1}$, respectively. The accuracy was within ranged from 90.4 to $104 \%$ and relative standard deviation (RSD) $(n=6)$ was less than $17.4 \%$.

Table 2. Linear range, correlation coefficient $(r)$, accuracy, and LLOQ $(n=6)$ employed in determination of the six compounds in rat plasma.

\begin{tabular}{|c|c|c|c|c|c|c|}
\hline Compounds & Regression Equation & $r$ & Linearity Range $\left(\mathrm{ng} \cdot \mathrm{mL}^{-1}\right)$ & LLOQ (ng.mL $\left.L^{-1}\right)$ & Accuracy (\%) & RSD (\%) \\
\hline Hyperin & $Y=0.00406 X+0.000488$ & 0.9996 & $1-250$ & 1 & 104 & 7.9 \\
\hline Chlorogenic acid & $Y=0.00113 X+0.00233$ & 0.9992 & $0.1-1500$ & 0.1 & 104 & 15 \\
\hline Neochlorogenic acid & $\mathrm{Y}=0.00134 \mathrm{X}+0.00254$ & 0.9990 & $4-1000$ & 4 & 102 & 4.6 \\
\hline P-coumaric acid & $Y=0.00366 X+0.00276$ & 0.9993 & $4-10000$ & 4 & 98.3 & 4.7 \\
\hline Astragalin & $\mathrm{Y}=0.00613 \mathrm{X}+0.000874$ & 0.9991 & $0.1-25$ & 0.1 & 90.4 & 17 \\
\hline Isoquercitrin & $Y=0.00286 X+0.000841$ & 0.9994 & $2-500$ & 2 & 102 & 10 \\
\hline
\end{tabular}

\subsubsection{Selectivity}

Selectivity was confirmed by determining the blank plasma from six different lots and comparing the MRM chromatographic profiles of plasma samples, which spiked with the six compounds. As shown in Figure 2, there was a good separation and no interference from endogenous components for all analytes. 


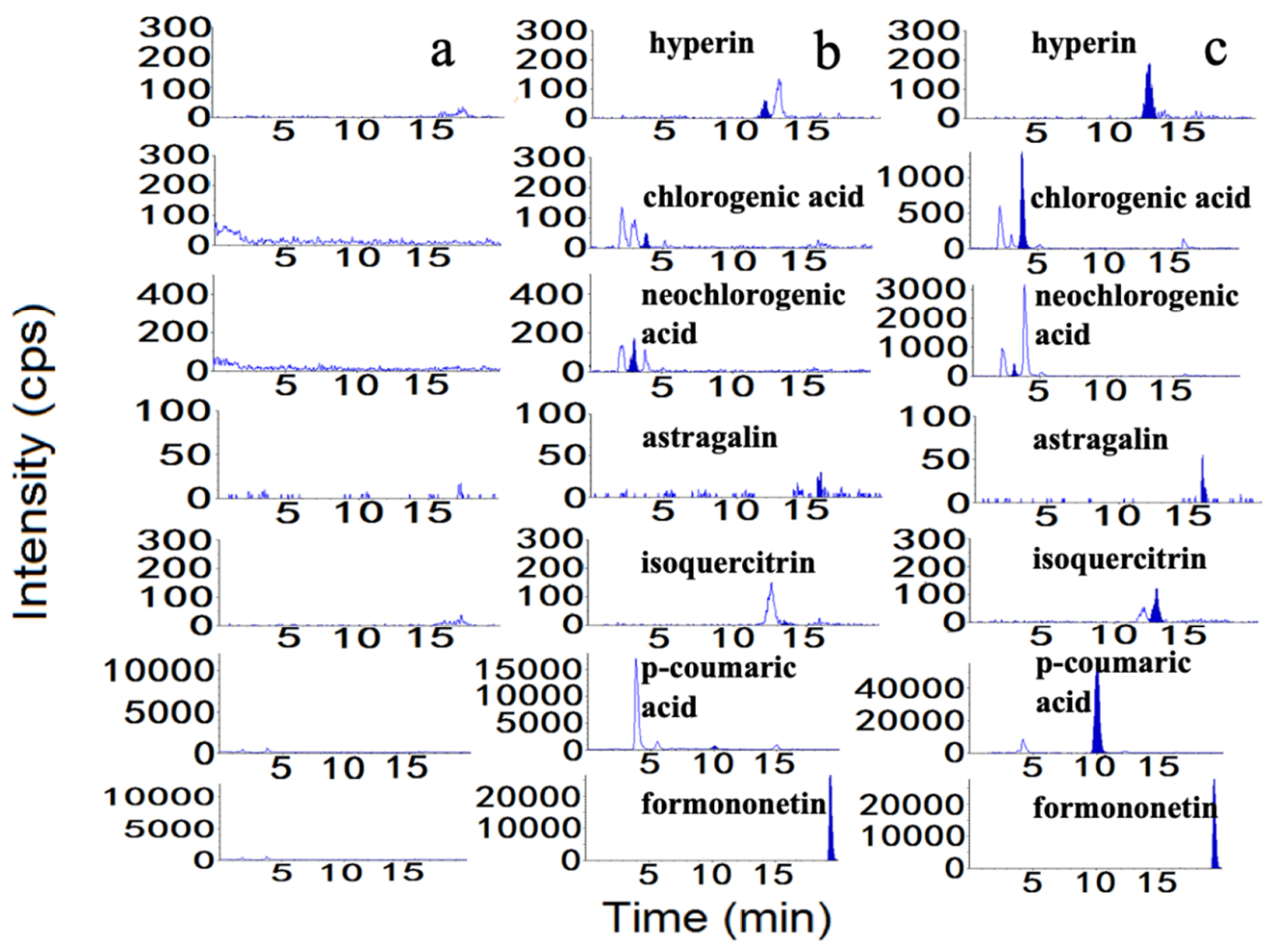

Figure 2. Representative chromatogram of (a) blank plasma, (b) blank plasma spiked with standard compounds at LLOQs, and (c) plasma sample.

\subsubsection{Accuracy and Precision}

As shown in Table 3, the precision was below $17.0 \%$ and the accuracy range of quality control (QC) samples was 80.3 to $114 \%$ at three levels for intra-day and inter-day values. It was demonstrated that the present method was reproducible and precise.

Table 3. Intra-day and inter-day values, recovery, matrix effect accuracy, and precision of six compounds $(n=6)$.

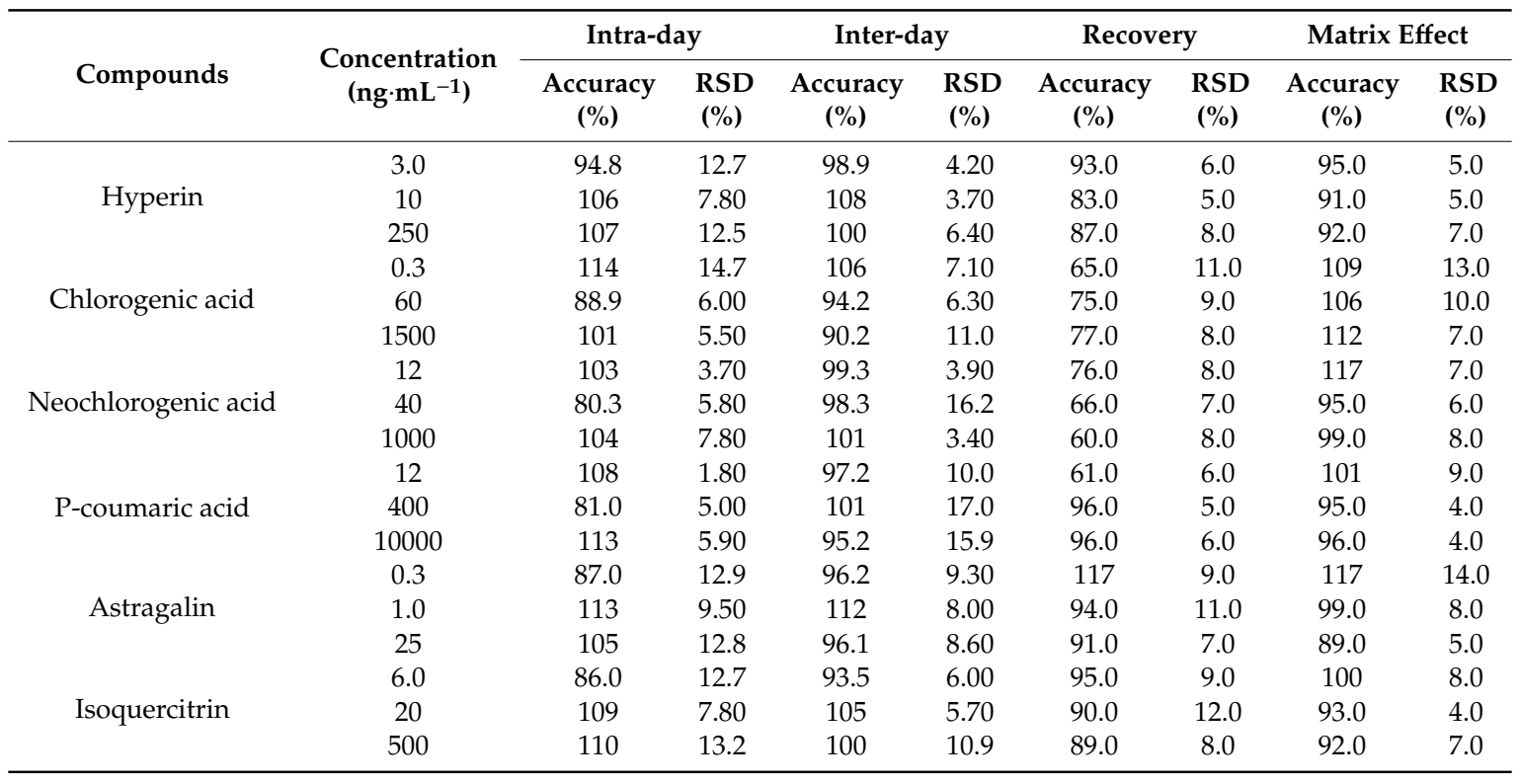




\subsubsection{Stability}

As shown in Table 4, the range of accuracy was 88.8 to $119 \%$, while the RSD was within $15.1 \%$ for the stock solution of analytes. This indicated that the six compounds were stable under conditions of three freeze-thaw, being kept in auto-sampler for $24 \mathrm{~h}$, and stored at $-80^{\circ} \mathrm{C}$ for 1 month.

Table 4. Stability of six compounds $(n=6)$.

\begin{tabular}{|c|c|c|c|c|c|c|c|}
\hline \multirow[b]{2}{*}{ Compounds } & \multirow{2}{*}{$\begin{array}{l}\text { Concentration } \\
\left(\mathrm{ng} \cdot \mathrm{mL}^{-1}\right)\end{array}$} & \multicolumn{2}{|c|}{ Freeze-Thaw Cycles } & \multicolumn{2}{|c|}{$-80^{\circ} \mathrm{C}$ for 1 Month } & \multicolumn{2}{|c|}{ Auto-Sampler for $24 \mathrm{~h}$} \\
\hline & & $\begin{array}{c}\text { Accuracy } \\
(\%)\end{array}$ & $\begin{array}{l}\text { RSD } \\
(\%)\end{array}$ & $\begin{array}{c}\text { Accuracy } \\
(\%)\end{array}$ & $\begin{array}{l}\text { RSD } \\
(\%)\end{array}$ & $\begin{array}{c}\text { Accuracy } \\
(\%)\end{array}$ & $\begin{array}{l}\text { RSD } \\
(\%)\end{array}$ \\
\hline \multirow{3}{*}{ Hyperin } & 3.0 & 104 & 14.8 & 103 & 6.20 & 90.2 & 13.6 \\
\hline & 10 & 116 & 10.7 & 92.5 & 3.80 & 107 & 13.2 \\
\hline & 250 & 107 & 12.0 & 102 & 3.90 & 100 & 10.8 \\
\hline \multirow{3}{*}{ Chlorogenic acid } & 0.3 & 107 & 13.1 & 104 & 6.70 & 94.4 & 12.0 \\
\hline & 60 & 119 & 12.6 & 105 & 13.5 & 115 & 13.9 \\
\hline & 1500 & 104 & 14.2 & 99.8 & 10.1 & 119 & 9.20 \\
\hline \multirow{3}{*}{ Neochlorogenic acid } & 12 & 99.9 & 12.4 & 103 & 12.3 & 98.9 & 13.3 \\
\hline & 40 & 102 & 8.30 & 103 & 14.3 & 115 & 9.00 \\
\hline & 1000 & 116 & 8.10 & 90.6 & 9.50 & 117 & 4.90 \\
\hline \multirow{3}{*}{ P-coumaric acid } & 12 & 98.8 & 10.4 & 91.5 & 12.7 & 88.8 & 15.1 \\
\hline & 400 & 112 & 8.30 & 100 & 11.9 & 116 & 8.30 \\
\hline & 10000 & 111 & 4.20 & 93.6 & 8.40 & 115 & 8.60 \\
\hline \multirow{3}{*}{ Astragalin } & 0.3 & 91.7 & 12.1 & 113 & 12.8 & 96.0 & 9.90 \\
\hline & 1.0 & 104 & 11.9 & 103 & 12.6 & 92.0 & 12.7 \\
\hline & 25 & 102 & 12.8 & 92.9 & 12.0 & 95.2 & 12.7 \\
\hline \multirow{3}{*}{ Isoquercitrin } & 6.0 & 107 & 11.7 & 106 & 12.1 & 111 & 11.5 \\
\hline & 20 & 109 & 14.2 & 91.4 & 13.2 & 103 & 8.00 \\
\hline & 500 & 114 & 14.9 & 99.1 & 8.90 & 104 & 8.00 \\
\hline
\end{tabular}

\subsubsection{Matrix Effects and Recoveries}

As listed in Table 3, the recoveries of the six compounds ranged from $60.0 \%$ to $117 \%$, while the RSD was below $12.0 \%$ at three concentration levels. Moreover, the matrix effects of the six compounds were in the range of $89.0 \%$ to $117 \%$, while the RSD was less than $14.0 \%$ at three concentrations.

\subsection{Pharmacokinetic Application}

After oral administration of the two processed products of CS, the only one that could not be tested (isoquercitrin) for the six compounds in rat plasma and the LLOQ reached $2 \mathrm{ng} \cdot \mathrm{mL}^{-1}$. The established method was used to investigate the pharmacokinetics of the five compounds after oral administration of SF-CS and SP-CS extracts. The pharmacokinetic profiles of five compound were represented in a one-compartment model. The mean plasma concentration time-curve outliers are illustrated in Figure 3. As shown in Table 5, the $\mathrm{AUC}_{(0-24 \mathrm{~h})}$ of p-coumaric (3198 \pm 635 and $\left.2567 \pm 792 \mathrm{ng} \cdot \mathrm{mL}^{-1}\right)$ was high in all compounds of the two processing procedures of CS, which indicated that it possessed abundant plasma exposure. The $\mathrm{AUC}_{(0-24 \mathrm{~h})}$ and $\mathrm{C}_{\max }$ of hyperin and astragalin were lower than those of other compounds in the two processing procedures of CS, which demonstrated that absorption of them was low in vivo. Moreover, the neochlorogenic acid and chlorogenic acid exhibited the double-peak phenomenon, which is related to the transformation among the phenolic acids.

Comparing the pharmacokinetic parameters of SF-CS and SP-CS (Figure 4 and Table 5), the $\mathrm{T}_{\max }$ of hyperin $(0.74 \pm 0.63 \mathrm{~h})$ after oral administration of SF-CS extract was longer than that of hyperin $(0.11 \pm$ $0.04 \mathrm{~h}$ ) after oral administration of SP-CS extract. The same trends for the other four components were found after oral administration of SF-CS and SP-CS extract. These results indicated that salt processing accelerated the absorption of the five compounds. Furthermore, the $\mathrm{C}_{\max }, \mathrm{AUC}_{0-24}$, and $\mathrm{AUC}_{0-\infty}$ of neochlorogenic acid of SF-CS have remarkable differences $(p<0.05$ for SP-CS, which shows that the absorption of neochlorogenic acid can be improved in SF-CS. Moreover, $\mathrm{AUC}_{(0-24 \mathrm{~h})}$ of astragalin was $1.27 \pm 0.76$ and $1.31 \pm 0.27 \mathrm{ng} \cdot \mathrm{mL}^{-1}$ for the two processing procedures of $C S$, respectively. The $C_{\max }$ of 
astragalin for SF-CS had significance differences ( $p<0.05$ with SP-CS). It was demonstrated that the plasma exposure of astragalin in CS was increased after salt processing. The present study indicated that salt processing could significantly increase the bioavailability of astragalin and accelerate the adsorption of hyperin, neochlorogenic acid, chlorogenic acid, and p-coumaric acid. This could be because salt processing increased the solubility of astragalin.

In order to explore the effect of salt in SP-CS on bioavailability and adsorption of five compounds in SF-CS, the pharmacokinetics of the five compounds after oral administration of SF-CS extract and SF-CS extract with salt were also performed by LC-MS/MS method. Comparing the pharmacokinetic parameters of SF-CS and SF-CS with salt (Figure 5), there were no differences between the main parameters of SF-CS and SF-CS with salt. It was indicated that the salt in SP-CS has no effects on the bioavailability and absorption of the five compounds for CS. However, salt processing significantly increased the bioavailability of astragalin and accelerated the adsorption of hyperin, neochlorogenic acid, chlorogenic acid, and p-coumaric acid. The reason might be that salt processing changed the composition proportions of herbal extract and pharmacokinetic interactions of multiple components in vivo. The presumption needs to be verified by the further research.
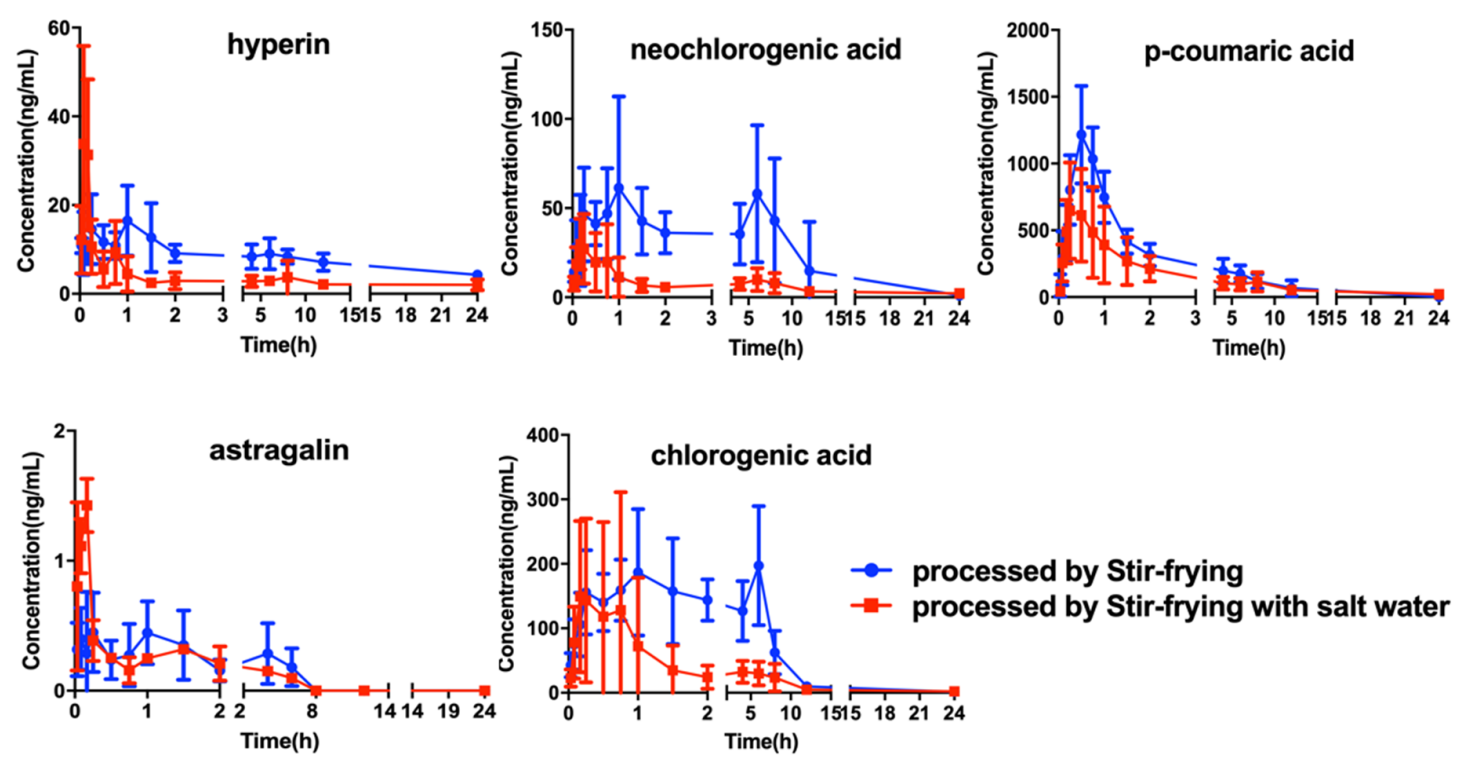

Figure 3. The mean plasma concentration time profiles of hyperin, neochlorogenic acid, chlorogenic acid, p-coumaric acid, and astragalin after oral administration of SF-CS and SP-CS ( $n=10$, mean \pm SD). 


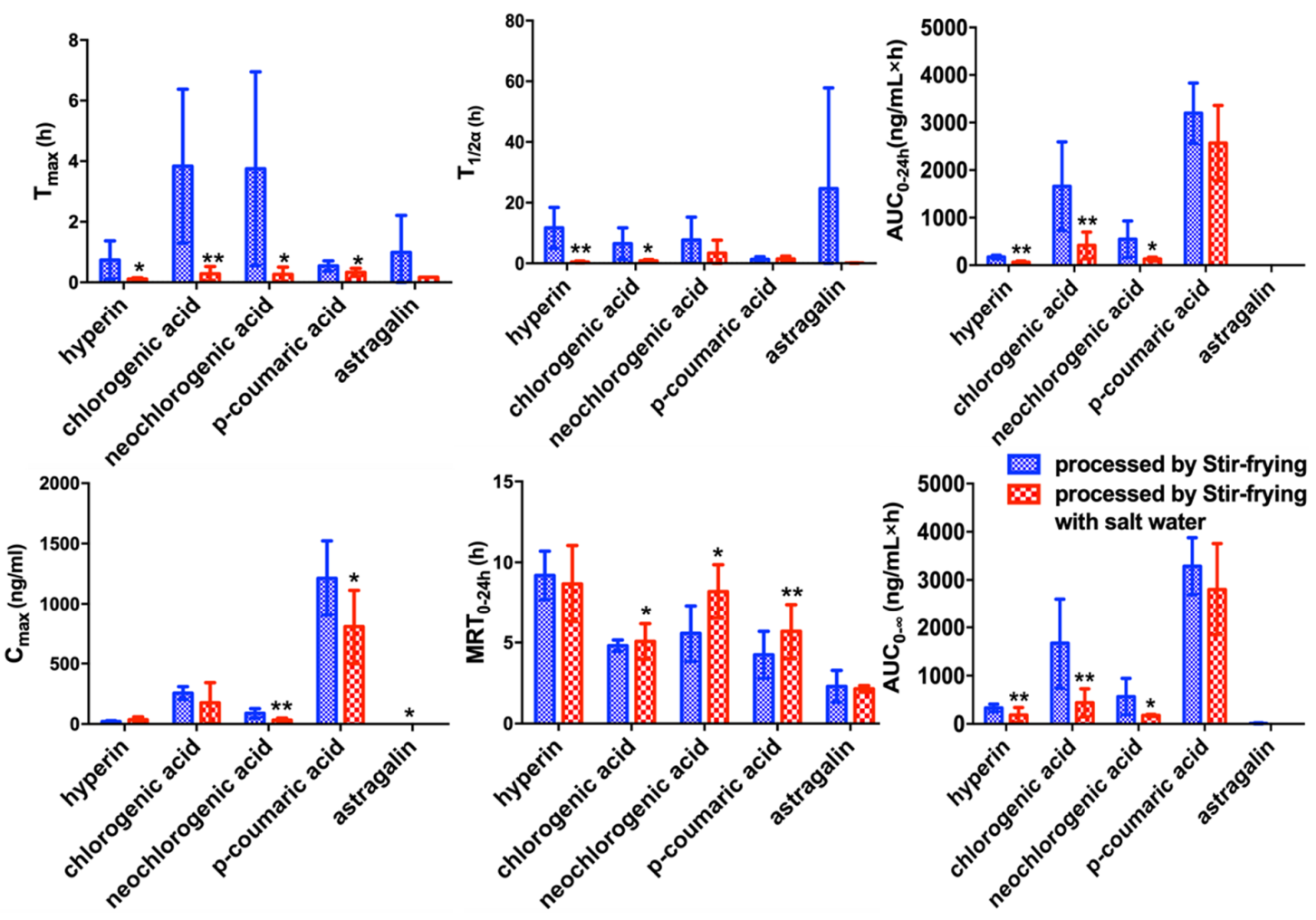

Figure 4. Pharmacokinetic comparison of oral administration of SF-CS and SP-CS extract $\left({ }^{*} p<0.05\right.$, ** $p<0.01)$.
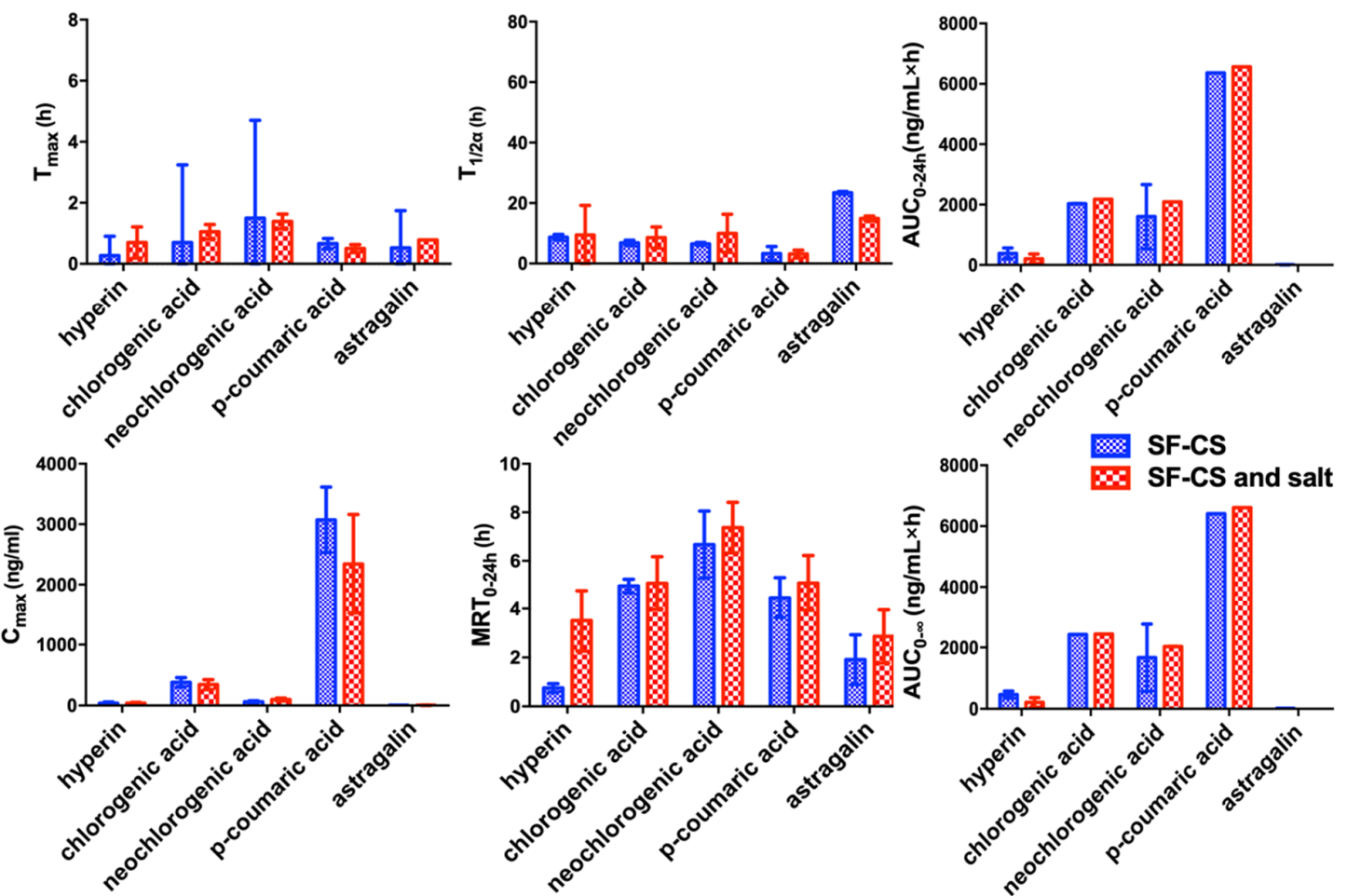

Figure 5. Pharmacokinetic comparison of oral administration of SF-CS extract and SF-CS extract and salt. 
Table 5. Pharmacokinetic parameters of six compounds after oral administration of SF-CS and SP-CS extract $(n=10$, mean \pm SD).

\begin{tabular}{|c|c|c|c|c|c|c|c|c|c|c|c|c|}
\hline \multirow{2}{*}{ Compound } & \multicolumn{2}{|c|}{ Hyperin } & \multicolumn{2}{|c|}{ Chlorogenic Acid } & \multicolumn{2}{|c|}{ Neochlorogenic Acid } & \multicolumn{2}{|c|}{ P-coumaric Acid } & \multicolumn{2}{|c|}{ Astragalin } & \multicolumn{2}{|c|}{ Isoquercitrin } \\
\hline & A & B & A & B & A & B & A & B & A & B & A & B \\
\hline dosage (mg/kg) & 21.8 & 27.5 & 31.1 & 49.0 & 6.09 & 6.64 & 0.59 & 0.87 & 1.87 & 1.30 & 1.98 & 1.99 \\
\hline $\mathrm{T}_{\max }(\mathrm{h})$ & 0.74 & 0.11 & 3.84 & 0.28 & 3.75 & 0.26 & 0.54 & 0.33 & 0.99 & 0.17 & & \\
\hline $\mathrm{C}_{\max }(\mathrm{ng} / \mathrm{mL})$ & $21.3 \pm 6.98$ & $36.6 \pm 21.9$ & $257 \pm 53$ & $175.2 \pm 167.7$ & $88.6 \pm 39.6$ & $31.8 \pm 17.3^{* *}$ & $1213 \pm 310$ & $805 \pm 307^{*}$ & $0.65 \pm 0.39$ & $1.43 \pm 0.21$ * & - & - \\
\hline $\mathrm{T}_{1 / 2 \mathrm{ka}}(\mathrm{h})$ & $2.15 \pm 4.21$ & $0.01 \pm 0.01$ & $0.63 \pm 0.87$ & $0.02 \pm 0.01$ & $1.12 \pm 1.66$ & $0.02 \pm 0.02$ & $0.15 \pm 0.12$ & $0.05 \pm 0.04$ & $2.27 \pm 5.13$ & $0.04 \pm 0.00$ & - & - \\
\hline $\mathrm{T}_{1 / 2 \alpha}(\mathrm{h})$ & $11.7 \pm 6.73$ & $0.46 \pm 0.30 * *$ & $6.53 \pm 5.20$ & $0.84 \pm 0.42 *$ & $7.72 \pm 7.50$ & $3.35 \pm 4.31$ & $1.32 \pm 0.86$ & $1.40 \pm 0.95$ & $24.6 \pm 33.2$ & $0.10 \pm 0.04$ & - & - \\
\hline $\mathrm{AUC}_{0-24 \mathrm{~h}}(\mathrm{ng} / \mathrm{mL} \cdot \mathrm{h})$ & $172 \pm 38$ & $63.0 \pm 27.8^{* *}$ & $1662 \pm 931$ & $417 \pm 281$ ** & $548 \pm 384$ & $129 \pm 38^{*}$ & $3198 \pm 635$ & $2567 \pm 792$ & $1.27 \pm 0.76$ & $1.31 \pm 0.27$ & - & - \\
\hline $\mathrm{AUC}_{0-\infty}(\mathrm{ng} / \mathrm{mL} \cdot \mathrm{h})$ & $331 \pm 80$ & $185 \pm 153 *$ & $1671 \pm 931$ & $438 \pm 289 * *$ & $567 \pm 376$ & $178 \pm 22 *$ & $3286 \pm 591$ & $2804 \pm 951$ & $11.2 \pm 10.8$ & $2.33 \pm 0.72$ & - & - \\
\hline $\mathrm{MRT}_{0-24 \mathrm{~h}}(\mathrm{~h})$ & $9.19 \pm 1.50$ & $8.66 \pm 2.37$ & $4.81 \pm 0.35$ & $5.06 \pm 1.10 *$ & $5.57 \pm 1.74$ & $8.18 \pm 1.66^{*}$ & $4.24 \pm 1.45$ & $5.68 \pm 1.69^{* *}$ & $2.30 \pm 0.98$ & $2.13 \pm 0.20$ & - & - \\
\hline
\end{tabular}

Note: A = SF-CS; B $=$ SP-CS; ${ }^{*} p<0.05, * * p<0.01$ 


\section{Materials and Methods}

\subsection{Chemicals and Reagents}

Reference substances of hyperin, neochlorogenic acid, chlorogenic acid, p-coumaric acid, astragalin, isoquercitrin, and formononetin (IS) with purity over 98.0\% were achieved from Chengdu Must Biotechnology Co., Ltd. (Chengdu, China). The acetonitrile and formic acid were obtained from Dikma (Foothill Ranch, CA, USA). Ultra-pure water was provided by Milli-Q system (Millipore, Boston, MA, USA).

\subsection{Apparatus and LC-MS/MS Conditions}

All components were measured on an Agilent 1200 HPLC system (Agilent Corporation, Santa Clara, CA, USA) and API 3200 triple quadrupole mass spectrometer (Concord, ON, Canada) in negative ion mode. Acetonitrile $(A)$ and $0.1 \%$ formic acid-water $(B)$ were used to separate the analytes on an Agilent Eclipse plus C18 column $(4.6 \mathrm{~mm} \times 100 \mathrm{~mm}, 1.8 \mu \mathrm{m})$ with a flow rate of $0.5 \mathrm{~mL} \cdot \mathrm{min}^{-1}$. The gradient program was as follows: $17 \% \mathrm{~A}(0-10 \mathrm{~min}), 17-48 \% \mathrm{~A}$ (10-12.5 $\left.\mathrm{min}\right)$, 48-52\% A (12.5-14 min), 52-54\% A (14-17 min), 54-60\% A (17-18.5 min), 60-90\% A (18.5-20 min). The equilibration time $(10 \mathrm{~min})$ of the sequence was added before the next injection. The column temperature was $30{ }^{\circ} \mathrm{C}$. The optimized main parameters, namely curtain gas, collision gas, ion Spray voltage, and temperature, were set at $30 \mathrm{psi}, 8 \mathrm{psi},-4000 \mathrm{~V}$, and $600{ }^{\circ} \mathrm{C}$, respectively. All of parameters are shown in Table 1.

\subsection{Preparation of SF-CS and SP-CS Extract}

The stir-frying and salt processing protocols for CS were performed according to the 0213 general rules recorded in the Chinese pharmacopeia 2015 edition [2].

The raw CS was heated with constant stirring until it became yellow with a slight crackle and light fragrance in a pot. Then, the SF-CS was poured out onto a plate to cool for the following experiment.

The raw CS was mixed well with salt water $(2 \mathrm{~g}$ of common salt was dissolved in $100 \mathrm{~mL}$ of water) until they were infused thoroughly in a closed vessel. Next, they were heated with constant stirring at $100-110^{\circ} \mathrm{C}$ for 4 min until they became yellow with a slight crackle and light fragrance [17]. Finally, the SP-CS was poured out onto a plate to cool for the following experiment.

SF-CS (1.5 kg) and SP-CS (1.5 kg) were accurately weighed, followed by addition of extraction solvent and refluxing twice independently (95\% ethanol the first time and 60\% ethanol the second time) with material-solvent ratio of 1:6 for $1 \mathrm{~h}$ each time. Under the reduced pressure, the extracted solutions were concentrated by using a rotary evaporator at $40{ }^{\circ} \mathrm{C}$ until dry. Extraction rates of SF-CS and SP-CS were $14.9 \%$ and $17.6 \%$, respectively.

\subsection{Preparation of Standard and Quality Control (QC) Samples}

Stock solutions $\left(1 \mathrm{mg} \cdot \mathrm{mL}^{-1}\right)$ were achieved by dissolving each standard substance in methanol. They were applied to obtain working solutions for calibration curves and QC samples by serial dilution. The IS solution was prepared in methanol at a concentration of $10 \mathrm{ng} \cdot \mathrm{mL}^{-1}$. All solutions were stored at $4{ }^{\circ} \mathrm{C}$.

\subsection{Plasma Samples Preparation}

The plasma sample $(100 \mu \mathrm{L})$, IS $(10 \mu \mathrm{L})$ and formic acid $(10 \mu \mathrm{L})$ were mixed and vortexed for $1 \mathrm{~min}$. Then, $1 \mathrm{~mL}$ of ethyl acetate was spiked into the mixed solution and centrifuged for $10 \mathrm{~min}$ at 14,000 rpm. The supernatant was transferred and evaporated with nitrogen gas. These residues were re-dissolved with $100 \mu \mathrm{L}$ methanol. The final solutions were vortexed for 2 min and centrifuged for $10 \mathrm{~min}$. The supernatant was injected into the HPLC-MS/MS and analyzed. 


\subsection{Method Validation}

The established method was evaluated following USFDA guideline [18]. The parameters contained sensitivity, accuracy, precision, recovery and so on.

\subsubsection{Linearity and LLOQ}

The calibration curve was performed by adding standard solutions into blank plasma. The concentration ranges of the standard solution were $0.1-1500,0.1-25,1-250,2-500,4-1000$, and $4-10,000 \mathrm{ng} \cdot \mathrm{mL}^{-1}$ for chlorogenic acid, astragalin, hyperin, isoquercitrin, neochlorogenic acid, and p-coumaric acid, respectively. The linearity was assessed with the peak area rate of the six compounds to IS concentration using $1 / \mathrm{X}^{2}$ weighting.

\subsubsection{Selectivity}

The selectivity was performed by analyzing the blank plasma sample, blank plasma added into the six compounds at the concentration of LLOQ, and real plasma orally administered with the SF-CS and SP-CS extracts.

\subsubsection{Accuracy and Precision}

The QC samples from six different batches at three levels (low, medium, and high) were applied to evaluate the accuracy and precision intra-day and over three different days. The assessed index was RSD and the percent ratios of the calculated concentration to nominal concentration. The range of accuracy should be 85.0 to $115 \%$.

\subsubsection{Stability}

The stabilities (including freeze-thaw cycles, auto-sampler and long-term stability) of all analytes were assessed with QC samples at three concentrations. All solutions were kept at $4{ }^{\circ} \mathrm{C}$.

\subsubsection{Matrix Effects and Recoveries}

The recovery and matrix effects of the six compounds were measured with six batches at three concentrations. The recovery was achieved by dividing the peak areas of the six compounds and IS added into plasma before extraction with those of the six compounds and IS added into processed plasma after extraction. The matrix effects were obtained by dividing results of the six compounds and IS added into processed plasma after extraction by those of the six compounds and IS in solvent. The values of recovery and matrix effects ranged from 85.0 to $115 \%$.

\subsection{Pharmacokinetic and Data Analysis}

The pharmacokinetic study was conducted in accordance with the Guidelines for the Care and Use of Laboratory Animals by USA National Institutes of Health and approved by the Animal Ethics Committee of Tianjin University of Traditional Chinese Medicine (the permit number: TCM-LAEC2019023, Tianjin, China). Male Sprague-Dawley rats (200-240 g) were fed in the animal laboratory of Tianjin University of Traditional Chinese Medicine under the standard conditions. These rats were fed and given water for 1 week before experimentation. Before administration of these extracts, all rats were fasted, although they were still given water until $12 \mathrm{~h}$ prior. The extraction yield of medicinal materials was influenced by the process. In order to investigate the influence of processing on the metabolic process of active ingredients in vivo, the same amount of raw CS was processed and administrated. Depending on the clinical dosage that was applied to the human body, the dosage of SF-CS extracts was $1.9 \mathrm{~g} \mathrm{~kg}^{-1}$ (an equivalent dosage of $12.75 \mathrm{~g} \mathrm{~kg}^{-1}$ raw CS) for oral administration, while extraction of SP-CS was $2.2 \mathrm{~g} \mathrm{~kg}^{-1}$ (an equivalent dosage of $12.50 \mathrm{~g} \mathrm{~kg}^{-1}$ raw CS). In order to investigate the influence of salt on the metabolic process of active ingredients in vivo, the dosage of SF-CS extracts was $1.9 \mathrm{~g} \mathrm{~kg}^{-1}$ (an equivalent dosage of $12.75 \mathrm{~g} \mathrm{~kg}^{-1}$ raw CS) and SF-CS 
extracts with salt $\left(0.26 \mathrm{~g} \mathrm{~kg}^{-1}\right)$ were used to perform the pharmacokinetic study. Plasma (about $250 \mu \mathrm{L}$ ) was gathered in $1.5 \mathrm{~mL}$ heparinized polythene tubes at $0,0.033,0.083,0.25,0.5,0.75,1,1.5,2,4$, $6,8,12$, and $24 \mathrm{~h}$ after oral administration of the two extracts. The collected plasma was centrifuged for $10 \mathrm{~min}$ at $6000 \mathrm{rpm}$ and stored at $-20{ }^{\circ} \mathrm{C}$ before analysis.

All of the pharmacokinetic parameters were estimated with the DAS1.0 system (Medical College of Wannan, China). The maximum concentration of oral administration of CS $\left(\mathrm{C}_{\max }\right)$ and the time to reach $C_{\max }\left(\mathrm{T}_{\max }\right)$ were achieved from the concentration-time profile. All values were shown as mean \pm standard deviation. Independent $t$-test was used to compare the pharmacokinetic parameters of the two processed CS.

\section{Conclusions}

A sensitive and efficient method was developed for simultaneous quantification of hyperin, chlorogenic acid, neochlorogenic acid, p-coumaric acid, and astragalin after oral administration of SF-CS and SP-CS extracts to rats. Moreover, the pharmacokinetic information revealed that salt processing accelerated the adsorption of hyperin, neochlorogenic acid, chlorogenic acid, and p-coumaric acid, and improved the bioavailability of astragalin. In conclusion, this investigation was established to assess pharmacokinetics of multiple orally administrated compounds of SF-CS and SP-CS extracts to rats. From a pharmacokinetic perspective, this can help us to understand the absorption of active compounds in vivo for two processed CS samples and will help in further study of SP-CS in clinical trials.

Author Contributions: Conceptualization, Y.C. and E.L.; data curation, J.L. (Jiao Liu), S.Z., J.L. (Jin Li) and Y.C.; formal analysis, J.L. (Jiao Liu), S.Z., J.L. (Jin Li), W.L., H.W. and J.H. (Jia Hao); funding acquisition, Y.C.; investigation, J.L. (Jiao Liu), W.L.; writing-original draft, S.Z., J.L. (Jin Li), X.G. and Y.C.; writing-review and editing, J.H. (Jun He).

Funding: This research was funded by Tianjin Research Program of Application Foundation and Advanced Technology (18JCYBJ95000), National Natural Science Foundation of China (81830112), Special Program of Talents Development for Excellent Youth Scholars in Tianjin and The APC was funded by Special Program of Talent Development for Excellent Youth Scholars in Tianjin in China.

Conflicts of Interest: The authors declare no conflict of interest.

\section{References}

1. Hajimehdipoor, H.; Kondori, B.M.; Amin, G.R.; Adib, N.; Rastegar, H.; Shekarchi, M. Development of a validated HPLC method for the simultaneous determination of compounds in Cuscuta chinensis Lam. by ultra-violet detection. DARU J. Fac. Pharm. 2012, 20, 57. [CrossRef] [PubMed]

2. Editorial Committee of the Pharmacopoeia of the People's Republic of China. Pharmacopoeia of the People's Republic of China; China Medical Science Press: Beijing, China, 2015.

3. Mavlonov, G.T.; Ubaidullaeva, K.A.; Kadryaeva, G.V.; Kuznetsova, N.N. Cytotoxic components of Cuscuta. Chem. Nat. Compd. 2008, 44, 409-410. [CrossRef]

4. Donnapee, S.; Li, J.; Yang, X.; Ge, A.H.; Donkor, P.O.; Gao, X.M.; Chang, Y.X. Cuscuta chinensis Lam.: A systematic review on ethnopharmacology, phytochemistry and pharmacology of an important traditional herbal medicine. J. Ethnopharmacol. 2014, 157, 292-308. [CrossRef] [PubMed]

5. Zhang, Y.; Xiong, H.; Xu, X.F.; Xue, X.; Liu, M.N.; Xu, S.Y.; Liu, H.; Gao, Y.; Zhang, H.; Li, X.R. Compounds identification in Semen Cuscutae by Ultra-High-Performance liquid chromatography (UPLCs) coupled to electrospray ionization mass spectrometry. Molecules 2018, 23, 1199. [CrossRef] [PubMed]

6. Ye, M.; Lee, S.G.; Chuang, E.S.; Lim, S.J.; Kim, W.S.; Yoon, H.; Kim, S.K.; Ahn, K.S.; Jang, Y.P.; Bae, H. Neuroprotective effects of cuscutae semen in a mouse modle of Parkinson's disease. Evid.-Based Complement. Altern. Med. 2014, 22, 150153.

7. Liao, J.C.; Chang, W.T.; Lee, M.S.; Chiu, Y.J.; Chao, W.J.; Lin, Y.C.; Lin, M.K.; Peng, Y.H. Antinociceptive and anti-flammatory activities of Cuscuta chinensis seeds in mice. Am. J. Chin. Med. 2014, 42, 223-242. [CrossRef] [PubMed] 
8. Han, S.X.; Yao, S.L.; Li, Y.Y.; Wang, T.S. Protective effect Cuscuta chinensis Lam. extract on myocardial ischemia/reperfusion injury in rats. Chin. Pharma. Bull. 2011, 27, 533-536.

9. Gao, F.X.; Zhou, C.; Qiu, W.Y.; Wu, H.W.; Li, J.; Peng, J.T.; Qiu, M.; Liang, C.; Gao, J.; Luo, S.P. Total compounds from Semen Cuscutae target MMP9 and promote invasion of EVT cells via Notch/AKT/MAPK signaling pathways. Sci. Rep. 2018, 8, 17342. [CrossRef] [PubMed]

10. Zhang, W.; Fu, Z.T.; Xie, Y.; Duan, Z.W.; Wang, Y.; Fan, R.H. High resolution UPLC-MS/MS method for simultaneous separation and determination of six compounds from Semen Cuscutae extract in rat plasma: Application to comparative pharmacokinetic studies in normal and kindey-deficient rats. Nat. Prod. Res. 2018, 30, 1-6.

11. Lin, M.K.; Yu, Y.L.; Chen, K.C.; Chang, W.T.; Lee, M.S.; Yang, M.J.; Cheng, H.C.; Liu, C.H.; Chen, D.C.; $\mathrm{Chu}, \mathrm{C} . \mathrm{L}$. Kaempferol from Semen cuscutae attenuates the immune function of dendritic cells. Immunobiology 2011, 216, 1103-1109. [CrossRef] [PubMed]

12. Chen, J.M. Enlightening Primer of Materia Medical; Traditional Chinese Medicine Ancient Books Press: Beijing, China, 1958.

13. Yang, S.; Xu, H.F.; Zhao, B.S.; Li, S.S.; Li, T.T.; Xu, X.F.; Zhang, T.J.; Lin, R.C.; Li, J.; Li, X.R. The difference of chemical components and biological activities of the crude product and salt-processed product from Semen Cuscutae. Evid.-Based Complement. Altern. Med. 2016, 16, 8656740. [CrossRef] [PubMed]

14. Zhang, L.; Ma, W.F.; Li, J.; He, J.; Zhang, P.; Zheng, F.; Zhang, B.L.; Gao, X.M.; Chang, Y.X. Influence of processing on pharmacokinetic of typical constituents in radix Polygoni multiflori after oral administration by LC-ESI-MS/MS. J. Ethnopharmacol. 2013, 148, 246-253. [CrossRef] [PubMed]

15. Zheng, C.; Mi, S.Q.; Luo, S.P.; Luo, Y.H. The determination of quercetin content by semen cuscutacae intragastric administration in rat and its pharmacokinetic study. Chin. Arch. Tradit. Chin. Med. 2004, 22, 1148-1150.

16. Peng, L.X. Take the Cuscuta as the Example, the Discussion Traditional Chinese Medicine Pharmacokinetic of Related Question Research. Ph.D. Thesis, Hubei University of Traditional Chinese Medicine, Wuhan, China, 2010; p. 97.

17. Xie, J. Study on Processing Technology and Standardization of Quality Standard of Cuscutae Semen (Tusizi). Master's Thesis, Liaoning University of Traditional Chinese Medicine, Shenyang, China, 2010; p. 27.

18. U.S. Department of Health and Human Services Food and Drug Administration. Guidance for Industry Bioanaly Method Validation. Fed. Regist. 2001, 66, 206-207.

Sample Availability: Samples of the compounds hyperin, chlorogenic acid, neochlorogenic acid, p-coumaric acid, astragalin, formononetin and isoquercitrin are available from the authors. 